\title{
Too WEIRD, Too Fast? Preprints about COVID-19 in psychology
}

This manuscript has not yet been peer-reviewed.

Arathy Puthillam

Department of Psychology, Monk Prayogshala, India

\section{Author Note}

Arathy Puthillam (iD) https://orcid.org/0000-0003-2426-8362

Arathy Puthillam, Senior Research Assistant, Department of Psychology, Monk Prayogshala

Correspondence concerning this article should be addressed to Arathy Puthillam, Department of Psychology, Monk Prayogshala (Sec 8), 4114, C Wing, Oberoi Garden Estates, Powai, Mumbai 400072, Maharashtra, India.

E-mail: ar@monkprayogshala.in

The study has been preregistered in an OSF repository and can be retrieved from here. 


\begin{abstract}
That behavioral sciences are overrepresented by some countries, in terms of samples and authors, is a well-documented finding. Considering the immediate policy implications, the present study aimed to scope whether this is true in the context of understanding the effects of the coronavirus as well. We assessed relevant preprints with "coronavirus" or "COVID-19" as keywords published on PsyArxiv between March-April, 2020, as well as between MayDecember, 2020 in terms of samples, participants, and authors. We found that some countries, such as the US, were overrepresented in both waves; papers based on authors from such countries, and employing samples from such countries were also more likely to be published in journals with higher impact factors, and were also more likely to be cited more. Implications, especially regarding a reductionist bifurcation of research as "WEIRD" or “non-WEIRD," are discussed.
\end{abstract}

Keywords: metascience, preprints, open science, sample diversity, researcher diversity, coronavirus, COVID-19 


\section{Too WEIRD, Too Fast: Preprints about COVID-19 in the psychological sciences}

Behavioral sciences are increasingly being used to inform policy across countries. Many practices from social and behavioral sciences have been extrapolated to understand how to change behaviors, including in the context of preventable infectious diseases, such as COVID-19.

However, most published and widely shared research in the behavioral sciences has been concentrated on a very small, homogeneous section of the population (e.g., Rad et al., 2018). As the COVID-19 pandemic spreads across the globe, many countries have relied on such research to communicate and use social norms (instead of hard laws) to nudge citizens to engage in hygiene practices. Interestingly, many researchers have also thrown their proverbial hat into the ring and have tried to assess, for example, how to communicate about the pandemic efficiently (e.g., Betsch, 2020; Lunn et al., 2020; Van Bavel et al., 2020).

In the case of a pandemic, where time and resources are scarce, and there is little empirical evidence for what definitely works and what does not, behavioral interventions might prove difficult. When time and resources are scarce, interpretations of policy could be biased (Mullainathan \& Shafir, 2013). This issue is also exacerbated by the fact that poor methods and statistics continue to plague science (Smaldino \& McElreath, 2016). No one is doubting the intentions of social and behavioral scientists, of course. However, there are many aspects of behavioral science that are circumspect. For instance, even in "normal" times, science is often conducted, interpreted, and disseminated by a homogenous group, from resource-heavy countries. Once exciting results are published and disseminated, the validity of such findings is rarely checked (but see Simonsohn et al., 2021). This is an enormous problem, and could possibly have disastrous consequences, especially during a global crisis. Instead of cross-validating their studies, many social scientists have collected 
data within short periods of time and have already written and published their results in some form or the other (Ruggeri et al., 2020), when ideally, the process takes months, if not years.

Rigorous academic work celebrates its objectivity. However, how can science be considered objective if it is interpreted by a handful of people (Meadon \& Spurrett, 2010) and overlooks a majority of the population? That research output about COVID-19 is heavily skewed has been previously discussed by a number of commentators. For instance, in economics, it is mostly senior male scientists who have been publishing pandemic-related working papers (Amano-Patiño et al., 2020). To check if the same holds true for psychology as well, we assessed papers published on PsyArxiv, a preprint repository. In other words, in the present set of studies, we ask: who is conducting and publishing results about COVID-19 in psychology? We do this using two waves of data. Wave 1 includes data from March and April, 2020; Wave 2 includes data till December, 2020. Specifically, we ask the following questions:

RQ1A. Who is conducting studies and publishing preprints about COVID-19?

B. Which of these preprints are being published, and in what journals?

RQ2A. Which samples are being used to study COVID-19 in psychology?

B. Which of these samples are being published, and in what journals?

RQs $1 \mathrm{~A}$ and 2A are particularly assessed for Wave 1.

\section{Sample Diversity in the Psychological Sciences}

Although psychology is supposed to be a study of human behavior, it is very limited in accurately capturing the human population. Arnett (2008) has shown that, in the top six journals published by the American Psychological Association, 68\% of the studies relied on US American samples, and 96\% relied on WEIRD samples. In the 2015-16 issues of Evolution \& Human Behaviour and Evolutionary Psychology, $81 \%$ of the samples were Western (Europe/North America/Australia), and 12\% was from Japan (Pollet \& Saxton, 
2019). In 2012, 65\% of the articles in Evolution \& Human Behaviour were WEIRD

(Kurzban, 2013). In the top five cross-cultural psychology journals, 64.6\% of samples and $96.7 \%$ of participants were WEIRD in $2015-2016 ; 33.03 \%$ of these samples were based in the US (Veillard, 2017). Between 2006 and 2010, articles published in three top (by impact factor) experimental developmental psychology journals, 57.65\% participants originated from the US, and $90.52 \%$ from WEIRD countries (Nielsen et al., 2017). In 2012, $96 \%$ of the papers published in Journal of Personality and Social Psychology were based on WEIRD samples (Kurzban, 2013).

\section{Researcher Diversity in Psychological Sciences}

When discussing issues of representation, it is also equally important to discuss who is producing this knowledge. Berry et al. (2002) have argued that psychology is culturebound and culture-blind. In other words, it is limited to concepts and origins from a small subpopulation of the world, and thus, does not accurately depict human behavior. It is also limited in that it often ignores the influence of culture in understanding human behavior (see also Berry, 2013). One potential way to mitigate this oversight may be by collaborating with researchers from non-WEIRD countries.

However, previous work has indicated that there is a lack of diversity in researchers as well, defined in terms of authors of published manuscripts (mostly first/primary authors). For instance, in developmental psychology journals, $61 \%$ were first-authored by those with institutional affiliations in the US, $20 \%$ in English-speaking countries, $15 \%$ in non-English speaking European countries, 4\% from Asia and Israel, and 2 articles had the first author located in Central or South America, but none in Middle East or Africa.

\section{The Present Study}


The present study, as discussed, aims to understand who is producing preprints about the coronavirus in the psychological sciences, and using which samples and populations. We do this by investigating the sample and participant characteristics of preprints on PsyArxiv as well as who authored these preprints. Data was collected in two waves: March-April, 2020 (i.e., within two months of the outbreak globally), and May-December, 2020.

However, bifurcating countries into WEIRD or non-WEIRD is reductionistic. For instance, how similar are US Americans and Cubans? Similarly, how similar are the Japanese and Indians? Technically, both groups would be clubbed together as "western" or "eastern." Further, how similar are rural US Americans, Asian Americans, and economically disadvantaged US Americans? Though Singapore and Japan are often considered nonWEIRD countries, are they comparable to smaller-scale societies? Thus, a WEIRD-nonWEIRD classification may be inaccurate and oversimplified (also see Ghai, 2021).

Considering this enormous limitation, in this study we present two lists: List 1 countries are those that are typically coded as "WEIRD," which includes the USA, Canada, the UK, Australia, and New Zealand. List 2 countries include those in List 1, but also Western European countries which are rich and democratic (Polity > 6; Marshall et al., 2017). Thus, a longer list of "WEIRD” countries was formed (Table S1).

Keeping in mind the limitations of this approach, for further clarity, we have also presented disaggregated results at the country-level. For instance, we present the number of samples, participants, and authors from each country in each wave of analysis.

\section{Method}

\section{Data and Code Availability}


Anonymized data and code for this paper are available on OSF at https://osf.io/4rfue/?view_only=3ac783758f334c4dae4b283c203631f5 (anonymous view only link)

\section{Wave 1 (March-April 2020)}

In attempting to understand the sampling and researcher diversity in preprints about coronavirus, two independent coders sourced papers published up to April 2020 on PsyArxiv, a preprint server for the psychological sciences in the first wave of data collection. To be clear, these papers have not been peer-reviewed and published in academic journals, as that process often takes months. However, preprints ensure rapid dissemination and visibility of research. At this stage, 40 preprints tagged "coronavirus" or "COVID-19" or "COVID" were sourced. These papers often represent sub-discipline diversity. Considering that, other than China, most countries detected their first cases between February and March 2020, an April deadline implies up to two months between conceptualization of the study and publication of the preprint.

The raters coded each manuscript for order of authorship (i.e., primary or secondary authors), the countries of authors' institutional affiliation, their gender (based on institutional or other reports of pronouns, if available), and the samples used. If there were multiple studies in a single paper, the sample characteristics from each study were checked separately. That is, if a paper comprised three studies, all recruiting US Americans, we counted each study separately. If the author or the sample was affiliated to more than one country, each country was coded. This is in line with previous studies (e.g., Arnett, 2008). Commentaries were excluded from analyses. Similarly, papers with no verifiable data about affiliation or sex of the authors were excluded from the analysis. Finally, forty papers were identified for analysis. A total of sixty-nine studies involving human participant samples were identified across the forty papers. 


\section{Wave 2 (May-December, 2020)}

Next, between March and May 2021, five coders traced each paper from Wave 1 to check which of them have been published and in which journals. Though imperfect, we used impact factors to assess the quality of journals, as done in previous studies.

Next, the coders sourced 597 preprints published on PsyArXiv between May and December, 2020 using the same protocol as in Wave 1. That is, commentaries, literature reviews, and other such papers without participants were excluded from analysis about samples or participants. However, online samples including data from Twitter were included in the analysis.

\section{Results}

\section{Wave 1}

\section{Sample diversity}

In the 65 studies, 21 countries were represented (Table 1A), and 14 studies included international samples or participants who were not disaggregated by country (Table $\mathrm{S} 1$ ). The number of disaggregated participants from each country ranged from 80 (Netherlands) to 250,855 (USA); the median number of participants was 540 .

Table 1A. Sample diversity in Wave 1

\begin{tabular}{lrrrrr}
\hline \multicolumn{5}{c}{$N$} & \multicolumn{3}{l}{ Percentages } \\
\hline Country & Participants & Samples & Participants & Participants Per \\
& Samples & & & & Sample \\
Austria & 1 & 540 & 1.96 & 0.19 & 540 \\
Canada & 3 & 1728 & 5.88 & 0.61 & 576 \\
China & 4 & 10776 & 7.84 & 3.80 & 2694 \\
Egypt & 1 & 540 & 1.96 & 0.19 & 540 \\
Italy & 1 & 2053 & 1.96 & 0.72 & 2053 \\
Japan & 1 & 540 & 1.96 & 0.19 & 540 \\
Kenya & 1 & 540 & 1.96 & 0.19 & 540 \\
Mexico & 1 & 540 & 1.96 & 0.19 & 540 \\
Netherlands & 1 & 80 & 1.96 & 0.03 & 80 \\
Nigeria & 1 & 540 & 1.96 & 0.19 & 540 \\
\hline
\end{tabular}




\begin{tabular}{lrrrrr}
\hline \multicolumn{5}{c}{$N$} & \multicolumn{3}{c}{ Percentages } & \\
\hline Norway & 1 & 4083 & 1.96 & 1.44 & 4083 \\
Poland & 2 & 454 & 3.92 & 0.16 & 227 \\
Qatar & 1 & 405 & 1.96 & 0.14 & 405 \\
Romania & 1 & 540 & 1.96 & 0.19 & 540 \\
Russia & 1 & 540 & 1.96 & 0.19 & 540 \\
South & 1 & 540 & 1.96 & 0.19 & 540 \\
Africa & & & & & 540 \\
South Korea & 1 & 540 & 1.96 & 0.19 & 540 \\
Sweden & 1 & 540 & 1.96 & 0.19 & 540 \\
Switzerland & 1 & 540 & 1.96 & 0.19 & 918.57 \\
UK & 7 & 6430 & 13.73 & 2.27 & 13202.89 \\
USA & 19 & 250855 & 37.25 & 88.53 & $\mathbf{5 5 5 5 . 7 6}$ \\
\hline \multicolumn{1}{c}{ Total } & $\mathbf{5 1}$ & $\mathbf{2 8 3 3 4 4}$ & $\mathbf{1 0 0}$ & $\mathbf{1 0 0}$ & \\
\hline
\end{tabular}

Researcher Diversity

A total of 181 countries were represented (including one case of no information; see Table 1B). Of these, 40 researchers $(22.10 \%)$ were based in the US, $12(6.62 \%)$ in the UK, 29 $(16.02 \%)$ in Germany, and $14(7.73 \%)$ in China.

\section{Publication status, citations, and impact factors}

By May 2021, one paper with a Netherlands-based primary author was cited 140 times, whereas on an average US, Canada, and Germany-based papers were cited more. Of the papers where primary authors were US-based, 7 were published, and 6 were not. On an average, papers with Europe-based primary authors were published in journals with higher impact factors (see Table 1B).

\begin{tabular}{lrrr}
\multicolumn{3}{c}{ Table 1B. Primary Authors' Countries of Affiliations (Wave 1) } \\
\hline Country & Not Published & Published & Total \\
\hline Canada & 2 & 1 & 3 \\
China & & 4 & 4 \\
Croatia & 2 & 1 & 1 \\
France & 12 & & 2 \\
Germany & & 1 & 13 \\
Italy & & 1 & 1 \\
Netherlands & 1 & & 1 \\
Norway & & 2 & 1 \\
Poland & & 1 & 1 \\
Slovakia & &
\end{tabular}




\begin{tabular}{lrrr}
\hline Country & Not Published & Published & Total \\
\hline Slovenia & & 1 & 1 \\
United Kingdom & 2 & 1 & 3 \\
United States & 6 & 7 & 13 \\
Grand Total & $\mathbf{2 5}$ & $\mathbf{2 1}$ & $\mathbf{4 6}$ \\
\hline
\end{tabular}

Median citations and impact factors across lists are presented in Table 1C. 
Table 1C. Median citations and impact factors (Wave 1)

\begin{tabular}{cccc}
\hline & & Citations & Impact \\
List 1 & Includes non-WEIRD countries & 23.5 & 2.685 \\
& Does not include non-WEIRD countries & 12 & 3.605 \\
List 2 & Includes non-WEIRD countries & 25 & 2.503 \\
& Does not include non-WEIRD countries & 12 & 3.454 \\
List 1 & & 33 & 2.294 \\
& Exclusively non-WEIRD & 16.5 & 3.605 \\
\hline
\end{tabular}

\section{Wave 2}

\section{Sample diversity}

A total of 614 papers had participants, and those were analyzed here. Next, $58.31 \%$ of the preprints had participants from List 1 countries, and $82.90 \%$ preprints had participants from List 2 countries. Further, $78.18 \%$ preprints included at least some participants from nonList 1 countries, such as India and Bangladesh. Sample data collected from before 2020 (e.g., longitudinal studies) were deleted from the analysis. A total of $36,909,784$ participants were employed in the various studies (range $=1$ to 3,24,00,000 participants; Mean=64079.49, Median=690.5).

The median number of participants from List 1 countries was 750, and 570 for those not from List 1; however, this was not a significant difference $\left(\mathrm{X}^{2}(1)=2.33, \mathrm{p}=.127\right)$. There was a significant difference $\left(\mathrm{X}^{2}(1)=13.364, \mathrm{p}<.001\right)$ in the median sample sizes of samples from List 2 countries. A country-wise break-up of samples is included in Table 2A.

Table 2A. Country-wise distribution of samples in Wave 2 preprints.

\begin{tabular}{|c|c|c|c|c|c|}
\hline Countries & Frequencies & $\mathrm{N}$ & Countries & Frequencies & $\mathrm{N}$ \\
\hline "At Home" & 1 & 1 & Ireland, UK, and USA & 1 & 234 \\
\hline $\begin{array}{l}\text { Australia, Brazil, } \\
\text { Canada, France, Germany, } \\
\text { Hong Kong, India, Japan, } \\
\text { Mexico, the United }\end{array}$ & 1 & 1411200 & Israel & 8 & 1878 \\
\hline
\end{tabular}




\begin{tabular}{|c|c|c|c|c|c|}
\hline Countries & Frequencies & $\mathrm{N}$ & Countries & Frequencies & $\mathrm{N}$ \\
\hline $\begin{array}{l}\text { Kingdom, and the } \\
\text { United States }\end{array}$ & & & & & \\
\hline 150 Countries & 1 & & Italy & 31 & 56378.09 \\
\hline 20 Countries & 1 & 38910 & Japan & 16 & 19343 \\
\hline 22 countries & 1 & 1759 & Kenya & 3 & 1050 \\
\hline 29 countries & 1 & 140 & $\begin{array}{l}\text { mainly Germany, } \\
\text { Switzerland and Austria }\end{array}$ & 1 & 2640 \\
\hline 47 Countries & 2 & 176362 & Malaysia & 1 & 895 \\
\hline 49 Countries & 1 & 50966 & Malta & 1 & 219 \\
\hline 55 countries & 1 & 25488 & Mexico & 1 & 102 \\
\hline 55 countries & 1 & 3593 & Middle East & 2 & 3 \\
\hline 6 countries & 1 & 348 & Middle East (incl. Israel) & 1 & 11 \\
\hline 69 Countries & 1 & 23629 & Nepal & 1 & 149 \\
\hline 8 Countries & 1 & 9889 & Netherlands & 17 & 10160 \\
\hline Africa & 1 & 2 & New Zealand & 12 & 7537 \\
\hline Argentina & 3 & 2388 & Nigeria & 3 & 2341 \\
\hline $\begin{array}{l}\text { Argentina, Australia, Brazil, } \\
\text { Canada, France, Germany, } \\
\text { Greece, Indonesia, Italy, } \\
\text { Japan, Netherlands, } \\
\text { Philippines, Romania, } \\
\text { Russia, Saudi Arabia, Serbia, } \\
\text { South Africa, South Korea, } \\
\text { Spain, Turkey, the United } \\
\text { Kingdom, Ukraine, and the } \\
\text { United States of America }\end{array}$ & 1 & 1000 & North America & 3 & 608 \\
\hline Armenia & 1 & 348 & Northern Ireland & 1 & 10 \\
\hline Asia & 3 & 46 & Norway & 10 & 46572 \\
\hline Asia (incl. India) & 1 & 5 & $\begin{array}{l}\text { Other European } \\
\text { Nationals }\end{array}$ & 2 & \\
\hline Australia & 23 & 13052 & Outside France & 1 & 55 \\
\hline Australia, Canada, UK, USA & 1 & 728 & Pakistan & 1 & 200 \\
\hline Australia/ New Zealand & 4 & 87 & Pan-America & 1 & 254 \\
\hline Austria & 6 & 583 & Peru & 2 & 446 \\
\hline Bangladesh & 3 & 2694 & Philippines & 2 & 1530 \\
\hline Belarus & 3 & 3674 & Poland & 29 & 28089 \\
\hline Belgium & 10 & 1929 & Portugal & 6 & 489 \\
\hline Bolivia & 1 & 242 & Republic of Ireland & 5 & 9883 \\
\hline Bosnia and Herzegovina & 4 & 1684 & Romania & 1 & 2704 \\
\hline Brazil & 8 & 26577 & Russia & 3 & 4419 \\
\hline Budapest & 1 & 77 & $\begin{array}{l}\text { Scotland, Wales and } \\
\text { Northern Ireland }\end{array}$ & 1 & 14 \\
\hline Bulgaria & 2 & 2413 & Scotland & 3 & 1511 \\
\hline California & 1 & 450 & Serbia & 2 & 445 \\
\hline Canada & 24 & 15607 & Siberia & 1 & 754 \\
\hline Canada, Ireland & 1 & 16 & Singapore & 1 & 505 \\
\hline China & 21 & 26988 & Slovakia & 1 & 783 \\
\hline Colombia & 3 & 1632 & South America & 3 & 25 \\
\hline Continental Europe & 1 & 400 & South Korea & 5 & 4296 \\
\hline Costa Rica & 2 & 2 & South-East Asia Region & 1 & 259 \\
\hline
\end{tabular}




\begin{tabular}{|c|c|c|c|c|c|}
\hline Countries & Frequencies & $\mathrm{N}$ & Countries & Frequencies & $\mathrm{N}$ \\
\hline Croatia & $\overline{8}$ & 4140 & Southern China & 1 & 152 \\
\hline Czech Republic & 8 & 12656 & Spain & 16 & 9090 \\
\hline Denmark & 15 & 81215 & Spain & 1 & 48 \\
\hline $\begin{array}{l}\text { Denmark, France, Hungary, } \\
\text { Italy, Sweden, UK, USA }\end{array}$ & 1 & & Sub-Sahara Africa & 3 & 23 \\
\hline $\begin{array}{l}\text { Eastern Mediterranean } \\
\text { Region }\end{array}$ & 1 & 459 & Sweden & 8 & 31705 \\
\hline Egypt & 1 & 96 & Switzerland & 15 & 15514 \\
\hline England & 6 & 1920 & Not specified & 11 & 415836 \\
\hline English respondents & 1 & 138 & Taiwan & 1 & 957 \\
\hline Estonia & 4 & 10 & Turkey & 6 & 6511 \\
\hline Ecuador & 1 & 249 & UAE & 1 & 1585 \\
\hline Europe & 4 & 1401 & UK & 71 & 354271 \\
\hline Europe and Russia & 2 & 2202 & UK / Ireland & 1 & 190 \\
\hline Europe or Eurasia & 1 & 202 & UK and Belgium & 1 & 340 \\
\hline Finland & 4 & 424 & UK and Northern Island & 1 & 11263 \\
\hline France & 24 & 36367 & $\begin{array}{l}\text { UK, Poland and other } \\
\text { EU countries }\end{array}$ & 1 & 497 \\
\hline Germany & 50 & 29106220 & Ukraine & 4 & 3405 \\
\hline $\begin{array}{l}\text { Germany, Netherlands, Italy, } \\
\text { Pakistan }\end{array}$ & 1 & 24 & Unknown & 1 & 4 \\
\hline $\begin{array}{l}\text { Germany, United Kingdom, } \\
\text { Ireland, Italy, and Pakistan }\end{array}$ & 1 & 43 & USA & 192 & 4291340 \\
\hline Global & 1 & 6675 & USA and India & 1 & 305 \\
\hline Greece & 5 & 4718 & USA and UK & 4 & 1159 \\
\hline Hong Kong & 4 & 2593 & Vietnam & 1 & 341 \\
\hline Hungary & 7 & 13797 & Wales & 1 & 8 \\
\hline India & 8 & 3481 & Western Pacific Region & 1 & 326 \\
\hline Indonesia & 6 & 3965 & (blank) & & 599 \\
\hline International (54 countries) & 1 & & & & \\
\hline Iran & 3 & 417 & & & \\
\hline Ireland & 7 & 4923 & & & \\
\hline Ireland, Scotland, Wales & 1 & 30 & & & \\
\hline
\end{tabular}

\section{Researcher Diversity}

A total of 3002 studies were coded, 17 of which did not have researcher countries identified. Of the remaining 2985, 2.24\% authors had institutional affiliations from multiple countries. Of the 2918 authors who were affiliated to a single country, $28.27 \%$ were affiliated to the US, 6.34\% to Germany and the UK each (an additional 5.82\% affiliated with England, $.71 \%$ to Scotland, $.03 \%$ to Wales, and $1.06 \%$ to Northern Ireland), and $5.14 \%$ were affiliated to the Netherlands. Table 2B represents all coded countries and Table S2A includes 
disaggregated results for authors with multi-country affiliations. Table S2B includes multicountry affiliations that are not disaggregated.

Table 2B. Countries represented in preprints between May and December 2020.

\begin{tabular}{|c|c|c|c|c|c|}
\hline $\begin{array}{l}\text { Country of Institutional } \\
\text { Affiliation }\end{array}$ & $\mathrm{N}$ & Percentage & $\begin{array}{l}\text { Country of Institutional } \\
\text { Affiliation }\end{array}$ & $\mathrm{N}$ & Percentage \\
\hline USA & 825 & 28.27 & Luxembourg & 10 & 0.34 \\
\hline Germany & 185 & 6.34 & Republic of Ireland & 10 & 0.34 \\
\hline UK & 185 & 6.34 & Portugal & 9 & 0.31 \\
\hline England & 170 & 5.83 & Croatia & 8 & 0.27 \\
\hline Netherlands & 150 & 5.14 & Pakistan & 8 & 0.27 \\
\hline Italy & 123 & 4.22 & UAE & 8 & 0.27 \\
\hline China & 111 & 3.80 & Finland & 6 & 0.21 \\
\hline Australia & 106 & 3.63 & Iran & 6 & 0.21 \\
\hline Poland & 106 & 3.63 & Korea & 6 & 0.21 \\
\hline Canada & 89 & 3.05 & Taiwan & 6 & 0.21 \\
\hline New Zealand & 58 & 1.99 & Bosnia and Herzegovina & 5 & 0.17 \\
\hline Switzerland & 54 & 1.85 & Slovakia & 5 & 0.17 \\
\hline France & 53 & 1.82 & Greece & 4 & 0.14 \\
\hline Denmark & 51 & 1.75 & Canada & 3 & 0.10 \\
\hline Norway & 46 & 1.58 & Colombia & 3 & 0.10 \\
\hline Ireland & 45 & 1.54 & Belgique & 2 & 0.07 \\
\hline Spain & 45 & 1.54 & Estonia & 2 & 0.07 \\
\hline Japan & 41 & 1.41 & Kenya & 2 & 0.07 \\
\hline Bangladesh & 37 & 1.27 & Peru & 2 & 0.07 \\
\hline Israel & 31 & 1.06 & Slovenia & 2 & 0.07 \\
\hline Northern Ireland & 31 & 1.06 & Vietnam & 2 & 0.07 \\
\hline Hungary & 29 & 0.99 & Bolivia & 1 & 0.03 \\
\hline India & 22 & 0.75 & Bosnia & 1 & 0.03 \\
\hline Scotland & 21 & 0.72 & Bulgaria & 1 & 0.03 \\
\hline Austria & 18 & 0.62 & Czechia & 1 & 0.03 \\
\hline Czech Republic & 18 & 0.62 & Ecuador & 1 & 0.03 \\
\hline Belgium & 17 & 0.58 & Jordon & 1 & 0.03 \\
\hline Brazil & 17 & 0.58 & Lithuania & 1 & 0.03 \\
\hline Serbia & 16 & 0.55 & Malaysia & 1 & 0.03 \\
\hline Turkey & 16 & 0.55 & Malta & 1 & 0.03 \\
\hline South Korea & 15 & 0.51 & Moldova & 1 & 0.03 \\
\hline Singapore & 14 & 0.48 & Sharjah & 1 & 0.03 \\
\hline Sweden & 14 & 0.48 & Slovak Republic & 1 & 0.03 \\
\hline Indonesia & 12 & 0.41 & South Africa & 1 & 0.03 \\
\hline Hong Kong & 11 & 0.38 & Thailand & 1 & 0.03 \\
\hline \multirow[t]{2}{*}{ Nigeria } & 11 & 0.38 & Ukraine & 1 & 0.03 \\
\hline & & & Wales & 1 & 0.03 \\
\hline
\end{tabular}


Further, 1592 authors (53\%) belonged to WEIRD countries, and 1410 (47\%) to nonWEIRD countries, based on List 1 countries. When List 2 is considered, 2593 (86.38\%) authors belonged to WEIRD countries, and 409 (13.62\%) to non-WEIRD countries.

\section{Publication status, citations, and impact factors}

By May 2021, 38.2\% preprints were published. Table 2C presents a country-level distribution of published and unpublished preprints. Out of the published preprints, $57.05 \%$ authors belonged to List 1 countries, and $87.89 \%$ to List 2 countries. Mean and median citations between primary authors from List 1 countries and those not from List 1 were not significantly different; however, the median citations were not significantly different between the two sets (Table 2D). On the other hand, mean and median citations for primary authors from List 2 countries was significantly higher than those not from List 2 countries (Table 2D).

Table 2C. Country-level distribution of published and unpublished papers

\begin{tabular}{|c|c|c|c|c|c|}
\hline $\begin{array}{l}\text { Country of } \\
\text { Institution }\end{array}$ & $\begin{array}{l}\text { Not } \\
\text { Published }\end{array}$ & Published & $\begin{array}{l}\text { Country of } \\
\text { Institution }\end{array}$ & $\begin{array}{l}\text { Not } \\
\text { Published }\end{array}$ & Published \\
\hline Australia & 52 & 54 & Lithuania & & 1 \\
\hline Australia, UK & 2 & & Luxembourg & 7 & 3 \\
\hline Australia, USA & 1 & & Malaysia & 1 & \\
\hline Austria & 14 & 4 & Malta & 1 & \\
\hline Austria, Canada & 1 & & Moldova & & 1 \\
\hline Austria, Switzerland & 2 & & No Information & 16 & 1 \\
\hline Bangladesh & 16 & 21 & Netherlands & 120 & 30 \\
\hline Bangladesh, UK & & 1 & $\begin{array}{l}\text { Netherlands, } \\
\text { Germany }\end{array}$ & 1 & \\
\hline Bangladesh, USA & & 1 & Netherlands, USA & 4 & \\
\hline Belgique & 2 & & New Zealand & 29 & 29 \\
\hline Belgium & 5 & 12 & New Zealand, Brazil & 1 & 1 \\
\hline Bolivia & & 1 & $\begin{array}{l}\text { New Zealand, } \\
\text { Germany }\end{array}$ & 2 & \\
\hline Bosnia & 1 & & Nigeria & 8 & 3 \\
\hline $\begin{array}{l}\text { Bosnia and } \\
\text { Herzegovina }\end{array}$ & 5 & & Northern Ireland & 21 & 10 \\
\hline Brazil & 9 & 8 & Northern Ireland, UK & 6 & 4 \\
\hline Bulgaria & 1 & & Norway & 33 & 13 \\
\hline Canada & 61 & 28 & Norway, Germany & & 1 \\
\hline Canada & 2 & 1 & Pakistan & 6 & 2 \\
\hline Canada, Sweden & 1 & 1 & Peru & & 2 \\
\hline China & 69 & 42 & Poland & 83 & 23 \\
\hline
\end{tabular}




\begin{tabular}{|c|c|c|c|c|c|}
\hline $\begin{array}{l}\text { Country of } \\
\text { Institution }\end{array}$ & $\begin{array}{l}\text { Not } \\
\text { Published }\end{array}$ & Published & $\begin{array}{l}\text { Country of } \\
\text { Institution }\end{array}$ & $\begin{array}{l}\text { Not } \\
\text { Published }\end{array}$ & Published \\
\hline Colombia & 2 & 1 & Poland, Germany & 1 & \\
\hline Croatia & 7 & 1 & Portugal & 9 & \\
\hline Czech Republic & 11 & 7 & Republic of Ireland & 10 & \\
\hline Czechia & 1 & & Scotland & 11 & 10 \\
\hline Denmark & 35 & 16 & Serbia & 3 & 13 \\
\hline Denmark, Netherlands & & 1 & Sharjah & 1 & \\
\hline Ecuador & & 1 & Singapore & 9 & 5 \\
\hline England & 103 & 67 & Slovak Republic & & 1 \\
\hline England, Australia & & 1 & slovakia & 2 & 3 \\
\hline England, Poland, Portuga & & 2 & Slovenia & & 2 \\
\hline Estonia & & 2 & South Africa & & 1 \\
\hline Finland & 6 & & South Korea & 15 & \\
\hline France & 36 & 17 & Spain & 25 & 20 \\
\hline $\begin{array}{l}\text { France, Italy, South } \\
\text { Korea }\end{array}$ & 1 & & Sweden & 12 & 2 \\
\hline Germany & 108 & 77 & Sweden, Australia & & 1 \\
\hline Germany, Estonia & & 1 & Switzerland & 30 & 24 \\
\hline $\begin{array}{l}\text { Germany, } \\
\text { Netherlands }\end{array}$ & 1 & & $\begin{array}{l}\text { Switzerland, Canada, } \\
\text { UK }\end{array}$ & 1 & \\
\hline Germany, Norway & & 1 & Switzerland, Poland & 1 & \\
\hline $\begin{array}{l}\text { Germany, } \\
\text { Switzerland }\end{array}$ & 1 & 3 & Taiwan & & 6 \\
\hline Germany, UK & 1 & 1 & Thailand & 1 & \\
\hline Germany, USA & 2 & & Turkey & 6 & 10 \\
\hline Greece & 3 & 1 & Turkey, UK & & 1 \\
\hline Hong Kong & 10 & 1 & UAE & 8 & \\
\hline Hungary & 20 & 9 & UK & 148 & 37 \\
\hline India & 13 & 9 & UK, Belgium & & 1 \\
\hline Indonesia & 12 & & UK, Italy & & 1 \\
\hline Iran & 5 & 1 & UK, Norway & 1 & \\
\hline Ireland & 23 & 22 & UK, USA & & 1 \\
\hline Israel & 22 & 9 & Ukraine & 1 & \\
\hline Israel, USA & & 1 & USA & 451 & 374 \\
\hline Italy & 60 & 63 & USA, Australia & 1 & 1 \\
\hline Italy, Spain & 1 & 1 & USA, Bangladesh & & 2 \\
\hline Japan & 26 & 15 & USA, Canada & & 2 \\
\hline Japan, Indonesia & 1 & & USA, Chile & 1 & \\
\hline Jordon & 1 & & USA, UK & 1 & \\
\hline Kenya & 2 & & Vietnam & 2 & \\
\hline Korea & 6 & & Wales & & 1 \\
\hline
\end{tabular}


Table 2D. Mean and median citations and impact factors

\begin{tabular}{|c|c|c|c|c|c|c|c|c|c|c|c|}
\hline & & & List 1 & & & & & List 2 & & & \\
\hline \multirow[b]{3}{*}{ Citations } & & $\mathrm{M}$ & SD & Median & $\bar{t}$ & $\mathrm{X} 2$ & $\mathrm{M}$ & $\begin{array}{l}\text { SD } \\
\end{array}$ & Median & $\mathrm{t}$ & $\mathrm{X} 2$ \\
\hline & WEIRD & 12.48 & 60.42 & 1 & -0.15 & 0.004 & 13.04 & 52.77 & 1 & $-2.24^{*}$ & $6.22^{* *}$ \\
\hline & $\begin{array}{l}\text { non- } \\
\text { WEIRD }\end{array}$ & 11.91 & 34.99 & 1 & & & 6.24 & 17.67 & 0 & & \\
\hline & WEIRD & 3.76 & 2.7 & 2.74 & $-2.25^{*}$ & $*$ & 3.56 & 2.42 & 2.74 & $-2.81^{* *}$ & $* *$ \\
\hline $\begin{array}{l}\text { Impact } \\
\text { Factors }\end{array}$ & $\begin{array}{l}\text { non- } \\
\text { WEIRD }\end{array}$ & 3.02 & 1.87 & 2.47 & & & 2.35 & 1.77 & 2.21 & & \\
\hline
\end{tabular}

\section{Discussion}

The current study assessed the researcher and sample diversity in preprints about COVID-19 in psychology, hosted on PsyArxiv. We found that countries such as Germany and the US had a greater number of researchers as well as samples and participants across time. In the first two months since COVID-19 became a pandemic (March-April 2020), a total of 65 studies were conducted and published as preprints, mostly with authors and samples from western and developed countries. Further, primary authors from these countries also showed higher median citations and published in journals with higher impact factors.

The preliminary analysis of papers published in the earlier stages of the pandemic is very much in line with previous studies that report an overreliance on samples from the US in published papers across the subdisciplines of psychology (e.g., Arnett, 2008; Veillard, 2017). It could be contended that there could have been opportunity costs for many researchers who could have spent their resources in other ways. However, that is the root of our argument this is true of researchers from the overrepresented countries as well. It is a known fact that often preliminary, novel findings are publicized and enter our collective imagination; any refutations or alternative findings, especially from other countries are thought to be endogenous to these "cultures" and are often not published in top journals (see Arnett, 2008; Rad et al., 2018). This exercise could be construed as a critique of fast science, in that we wanted to explore to what extent preliminary findings are published fast, and who is 
publishing them. This is also in line with what we found with respect to citations and journal impact factors of preprints that were published.

In the next stages of the pandemic (May-December 2020), a similar trend was observed with respect to citations as well as impact factors, in that, authors from western, developed countries, including France and Germany, had published in "top" journals, and were cited more. Further, there was also a higher number of samples as well as median sample sizes from western, developed countries. This shows the overreliance of samples as well as trust in science by authors from such countries, which is not necessarily dependent on who is producing preprints.

This is also in line with the general finding that psychology is dominated by authors from the US. It has been argued before that when applying such science to policies, caution needs to be exercised. Particularly, this also affects generalizability of theories in the psychological sciences (IJzerman et al., 2020, 2021).

It is also important to note that researchers in developing countries often face issues relevant to the nascency of the scientific process. For instance, research infrastructure is often underdeveloped and research funds are limited (see Onie, 2020). These exacerbate the problems with understanding human behavior more broadly. For instance, without specific measures and theories that are not invariant across countries, it may be difficult for researchers from countries where psychological science has not yet developed to operate. A related problem is also in studying constructs in a culture-blind manner (Berry et al., 2002), wherein even though constructs may not be culture-blind, they are assumed to be so based on previous work focusing on specific countries such as the US. Similarly, constructs not relevant to the "mainstream" frameworks of psychology, such as caste in India, are not studied. Further, underrepresented or Eastern countries East are usually studied as absolutes, 
which is also reductionistic. In other words, a narrow lens, for example, based on researchers in the US, is usually employed to study other countries.

In this light, it is also important to note the limitations of this study. First, though authors, samples, and participants were assessed, we were unable to assess the constructs, and whether there was diversity in the constructs studied. For example, were authors from countries with indigenous populations able to assess constructs specific to such populations. Relatedly, we were unable to assess whether there were within-country representation across countries. Further, many preprints did not indicate specific countries that were sampled, reducing accuracy, indicating that better standards for reporting of samples in psychological sciences are required. Next, only COVID-19-related manuscripts were assessed. It is possible that authors from other countries did not focus on research related to the coronavirus. This is in line with other issues such as a lack of research infrastructure and funding in some countries, which could have made it more difficult for these researchers to conduct research related to coronavirus in such a short span. Further, we only concentrated on preprints in English, and could not consider those in other languages. Future research should attempt to assess languages other than English. Next, we only assessed preprints published on PsyArxiv. It is possible that only certain researchers from some countries have started putting up their working papers onto a repository such as PsyArxiv. Future work may consider papers that are peer-reviewed and published, considering that not every paper is published as a working paper due to various institutional and grant-related constraints. We assessed journal quality and preprint impact using citations and impact factors, both of which are imperfect metrics. Future research could assess which preprints or published papers are used to create policies at local, national, and global levels.

Where, then, does this leave us? If psychological science is needed to inform policy, it is imperative that the science itself is unshakable. This is especially true in the case of 
immediate danger, as seems to be the case with the pandemic. If most researchers concentrate on collecting data from a few countries, and derive insights based on restricted sampling, scientific objectivity is questionable. It is also vital that researchers collect data about the countries and ethnicities of their participants, so that constraints on generalizability are clearer.

\section{Conclusion}

The present study assessed preprints published on PsyArXiv in two waves: immediately after the onset of the pandemic across the world, and between two and ten months after its onset. We found that in the first wave, there was an overrepresentation of authors and samples from the US and Germany. In the second wave, though more geographical diversity was found, authors and samples from developed and European countries were overrepresented. 


\section{References}

Amano-Patiño, N., Faraglia, E., Giannitsarou, C., \& Hasna, Z. (2020, May 2). Who is doing new research in the time of COVID-19? Not the female economists. VoxEU.Org. https://voxeu.org/article/who-doing-new-research-time-covid-19-not-femaleeconomists

Arnett, J. J. (2008). The neglected 95\%: Why American psychology needs to become less American. The American Psychologist, 63(7), 602-614. https://doi.org/10.1037/0003066X.63.7.602

Berry, J. W. (2013). Global psychology. South African Journal of Psychology, 43(4), 391401. https://doi.org/10.1177/0081246313504517

Berry, J. W., Berry, J. W., Poortinga, Y. H., Segall, M. H., \& Dasen, P. R. (2002). Crosscultural psychology: Research and applications. Cambridge University Press.

Betsch, C. (2020). How behavioural science data helps mitigate the COVID-19 crisis. Nature Human Behaviour, 4(5), 438-438. https://doi.org/10.1038/s41562-020-0866-1

Ghai, S. (2021). It's time to reimagine sample diversity and retire the WEIRD dichotomy. Nature Human Behaviour, 5(8), 971-972. https://doi.org/10.1038/s41562-021-011759

IJzerman, H., Dutra, N., Silan, M., Adetula, A., Forscher, P. S., \& Basnight-Brown, D. (2021, August 30). Psychological Science Needs the Entire Globe. Observer. https://www.psychologicalscience.org/observer/global-psych-science

IJzerman, H., Lewis, N. A., Przybylski, A. K., Weinstein, N., DeBruine, L., Ritchie, S. J., Vazire, S., Forscher, P. S., Morey, R. D., Ivory, J. D., \& Anvari, F. (2020). Use 
caution when applying behavioural science to policy. Nature Human Behaviour, 4(11), 1092-1094. https://doi.org/10.1038/s41562-020-00990-w

Kurzban, R. (2013, September 25). Is Evolutionary Psychology WEIRD or NORMAL? Evolutionary Psychology Blog Archive. https://web.sas.upenn.edu/kurzbanepblog/2013/09/25/is-evolutionary-psychologyweird-or-normal/

Lunn, P., Belton, C., Lavin, C., McGowan, F., Timmons, S., \& Robertson, D. (2020). Using behavioural science to help fight the coronavirus. https://www.esri.ie/publications/using-behavioural-science-to-help-fight-thecoronavirus

Marshall, M. G., Gurr, T. R., \& Jaggers, K. (2017). Polity IV Project: Political Regime Characteristics and Transitions, 1800-2016 (Polity IV Project). Center for Systemic Peace. https://www.systemicpeace.org/inscr/p4manualv2016.pdf

Meadon, M., \& Spurrett, D. (2010). It's not just the subjects - there are too many WEIRD researchers. Behavioral and Brain Sciences, 33(2-3), 104-105. https://doi.org/10.1017/S0140525X10000208

Mullainathan, S., \& Shafir, E. (2013). Decision making and policy in contexts of poverty. The Behavioral Foundations of Public Policy, 281-297.

Nielsen, M., Haun, D., Kärtner, J., \& Legare, C. H. (2017). The persistent sampling bias in developmental psychology: A call to action. Journal of Experimental Child Psychology, 162, 31-38. https://doi.org/10.1016/j.jecp.2017.04.017 
Onie, S. (2020). Redesign open science for Asia, Africa and Latin America. Nature, 587(7832), 35-37. https://doi.org/10.1038/d41586-020-03052-3

Pollet, T. V., \& Saxton, T. K. (2019). How Diverse Are the Samples Used in the Journals 'Evolution \& Human Behavior' and 'Evolutionary Psychology’? Evolutionary Psychological Science, 5(3), 357-368. https://doi.org/10.1007/s40806-019-00192-2

Rad, M. S., Martingano, A. J., \& Ginges, J. (2018). Toward a psychology of Homo sapiens: Making psychological science more representative of the human population. Proceedings of the National Academy of Sciences, 115(45), 11401-11405. https://doi.org/10.1073/pnas.1721165115

Ruggeri, K., van der Linden, S., Wang, C., Papa, F., Afif, Z., Riesch, J., \& Green, J. (2020, May 23). Standards for evidence in policy decision-making. Behavioural and Social Sciences at Nature Research. http://socialsciences.nature.com/users/399005-kairuggeri/posts/standards-for-evidence-in-policy-decision-making

Simonsohn, U., Nelson, L., \& Simmons, J. (2021, August 17). Evidence of Fraud in an Influential Field Experiment About Dishonesty. Data Colada. https://datacolada.org/98

Smaldino, P. E., \& McElreath, R. (2016). The natural selection of bad science. Royal Society Open Science, 3(9), 160384. https://doi.org/10.1098/rsos.160384

Van Bavel, J. J., Baicker, K., Boggio, P. S., Capraro, V., Cichocka, A., Cikara, M., Crockett, M. J., Crum, A. J., Douglas, K. M., Druckman, J. N., Drury, J., Dube, O., Ellemers, N., Finkel, E. J., Fowler, J. H., Gelfand, M., Han, S., Haslam, S. A., Jetten, J., ... Willer, R. (2020). Using social and behavioural science to support COVID-19 
pandemic response. Nature Human Behaviour, 4(5), 460-471.

https://doi.org/10.1038/s41562-020-0884-Z

Veillard, N. (2017). WEIRD sampling in cross-cultural psychology, should it not be less WEIRD and more representative? The overrepresentation of individuals from Western educated industrialized and democratic countries as sample populations in cross-cultural psychology research [Master's Thesis, Leiden University]. Leiden University Student Repository. https://studenttheses.universiteitleiden.n1/handle/1887/56361 


\section{Contributions}

AP contributed to the conception, design, analysis and interpretation of data, as well as drafting of the manuscript.

\section{Acknowledgements}

The author acknowledges the coders who assisted in collecting data, as well as HK, who helped with proofreading.

\section{Funding information}

The author(s) received no particular financial support for the research, authorship, and/or publication of this article.

\section{Competing Interests}

No particular competing interests. However, the author resides and works in a "non-WEIRD" country.

\section{Data Accessibility Statement}

Data and code linked to this manuscript is openly available here. 


\section{Supplementary Tables}

Table S1A. Countries in List 2

\begin{tabular}{|c|c|}
\hline Countries & \\
\hline Czech Republic & Lithuania \\
\hline Australia $^{*}$ & Luxembourg \\
\hline Austria & Malta \\
\hline Belgique & Moldova \\
\hline Belgium & Netherlands \\
\hline Bosnia and Herzegovina & New Zealand \\
\hline Bulgaria & Northern Ireland ${ }^{*}$ \\
\hline Canada & Norway \\
\hline Croatia & Poland \\
\hline Czech Republic & Portugal \\
\hline Czechia & Republic of Ireland \\
\hline Denmark & Scotland $^{*}$ \\
\hline England $^{*}$ & Serbia \\
\hline Estonia & Slovak Republic \\
\hline Finland & Slovakia \\
\hline France & Slovenia \\
\hline Germany & Spain \\
\hline Germany & Sweden \\
\hline Greece & Switzerland \\
\hline Hungary & $U K^{*}$ \\
\hline Ireland & $U S^{*}$ \\
\hline Italy & Wales $^{*}$ \\
\hline
\end{tabular}

${ }^{*}$ Countries in italics and marked with an asterisk are included in List 1 as well as List 2 
Table S2A. Disaggregated list of countries for authors with multi-country affiliations.

\begin{tabular}{|c|c|c|c|c|c|}
\hline Country & $\mathbf{N}$ & $\%$ & Country & $\mathbf{N}$ & $\%$ \\
\hline US & 841 & 27.448 & Portugal & 11 & 0.359 \\
\hline UK & 205 & 6.691 & Luxembourg & 10 & 0.326 \\
\hline Germany & 201 & 6.56 & Republic of Ireland & 10 & 0.326 \\
\hline England & 173 & 5.646 & Croatia & 8 & 0.261 \\
\hline Netherlands & 157 & 5.124 & Pakistan & 8 & 0.261 \\
\hline Italy & 127 & 4.145 & UAE & 8 & 0.261 \\
\hline China & 111 & 3.623 & Finland & 6 & 0.196 \\
\hline Australia & 110 & 3.59 & Iran & 6 & 0.196 \\
\hline Poland & 110 & 3.59 & Korea & 6 & 0.196 \\
\hline Canada & 95 & 3.101 & Taiwan & 6 & 0.196 \\
\hline New Zealand & 62 & 2.023 & $\begin{array}{l}\text { Bosnia and } \\
\text { Herzegovina }\end{array}$ & 5 & 0.163 \\
\hline Switzerland & 62 & 2.023 & Slovakia & 5 & 0.163 \\
\hline France & 54 & 1.762 & Greece & 4 & 0.131 \\
\hline Denmark & 52 & 1.697 & Canada & 3 & 0.098 \\
\hline Norway & 49 & 1.599 & Colombia & 3 & 0.098 \\
\hline Spain & 47 & 1.534 & Estonia & 3 & 0.098 \\
\hline Ireland & 45 & 1.469 & Belgique & 2 & 0.065 \\
\hline Japan & 42 & 1.371 & Kenya & 2 & 0.065 \\
\hline Bangladesh & 41 & 1.338 & Peru & 2 & 0.065 \\
\hline $\begin{array}{l}\text { Northern } \\
\text { Ireland }\end{array}$ & 41 & 1.338 & Slovenia & 2 & 0.065 \\
\hline Israel & 32 & 1.044 & Bolivia & 1 & 0.033 \\
\hline Hungary & 29 & 0.946 & Bosnia & 1 & 0.033 \\
\hline India & 22 & 0.718 & Bulgaria & 1 & 0.033 \\
\hline Austria & 21 & 0.685 & Chile & 1 & 0.033 \\
\hline Scotland & 21 & 0.685 & Czechia & 1 & 0.033 \\
\hline Brazil & 19 & 0.62 & Ecuador & 1 & 0.033 \\
\hline Belgium & 18 & 0.587 & Jordon & 1 & 0.033 \\
\hline $\begin{array}{l}\text { Czech } \\
\text { Republic }\end{array}$ & 18 & 0.587 & Lithuanina & 1 & 0.033 \\
\hline Turkey & 18 & 0.587 & Malaysia & 1 & 0.033 \\
\hline NA & 17 & 0.555 & Malta & 1 & 0.033 \\
\hline Sweden & 17 & 0.555 & Moldova & 1 & 0.033 \\
\hline Serbia & 16 & 0.522 & Sharjah & 1 & 0.033 \\
\hline South Korea & 16 & 0.522 & Slovak Republic & 1 & 0.033 \\
\hline Singapore & 14 & 0.457 & South Africa & 1 & 0.033 \\
\hline Indonesia & 13 & 0.424 & Thailand & 1 & 0.033 \\
\hline Hong Kong & 11 & 0.359 & Ukraine & 1 & 0.033 \\
\hline Nigeria & 11 & 0.359 & & & \\
\hline
\end{tabular}


Table S2B. List of multi-country affiliations for authors in List 2

\begin{tabular}{|c|c|c|c|c|c|}
\hline $\begin{array}{l}\text { Country of } \\
\text { Institutional } \\
\text { Affiliation }\end{array}$ & $\mathrm{N}$ & Percentage & $\begin{array}{l}\text { Country of Institutional } \\
\text { Affiliation }\end{array}$ & $\mathrm{N}$ & Percentage \\
\hline USA & 825 & 27.48 & Belgique & 2 & 0.07 \\
\hline Germany & 185 & 6.16 & Canada, Sweden & 2 & 0.07 \\
\hline UK & 185 & 6.16 & $\begin{array}{l}\text { England, Poland, } \\
\text { Portugal }\end{array}$ & 2 & 0.07 \\
\hline England & 170 & 5.66 & Estonia & 2 & 0.07 \\
\hline Netherlands & 150 & 5 & Germany, UK & 2 & 0.07 \\
\hline Italy & 123 & 4.1 & Germany, USA & 2 & 0.07 \\
\hline China & 111 & 3.7 & Italy, Spain & 2 & 0.07 \\
\hline Australia & 106 & 3.53 & Kenya & 2 & 0.07 \\
\hline Poland & 106 & 3.53 & New Zealand, Brazil & 2 & 0.07 \\
\hline Canada & 89 & 2.96 & New Zealand, Germany & 2 & 0.07 \\
\hline New Zealand & 58 & 1.93 & Peru & 2 & 0.07 \\
\hline Switzerland & 54 & 1.8 & Slovenia & 2 & 0.07 \\
\hline France & 53 & 1.77 & USA, Australia & 2 & 0.07 \\
\hline Denmark & 51 & 1.7 & USA, Bangladesh & 2 & 0.07 \\
\hline Norway & 46 & 1.53 & USA, Canada & 2 & 0.07 \\
\hline Ireland & 45 & 1.5 & Vietnam & 2 & 0.07 \\
\hline Spain & 45 & 1.5 & Australia, USA & 1 & 0.03 \\
\hline Japan & 41 & 1.37 & Austria, Canada & 1 & 0.03 \\
\hline Bangladesh & 37 & 1.23 & Bangladesh, UK & 1 & 0.03 \\
\hline Israel & 31 & 1.03 & Bangladesh, USA & 1 & 0.03 \\
\hline Northern Ireland & 31 & 1.03 & Bolivia & 1 & 0.03 \\
\hline Hungary & 29 & 0.97 & Bosnia & 1 & 0.03 \\
\hline India & 22 & 0.73 & Bulgaria & 1 & 0.03 \\
\hline Scotland & 21 & 0.7 & Czechia & 1 & 0.03 \\
\hline Austria & 18 & 0.6 & Denmark, Netherlands & 1 & 0.03 \\
\hline Czech Republic & 18 & 0.6 & Ecuador & 1 & 0.03 \\
\hline Belgium & 17 & 0.57 & England, Australia & 1 & 0.03 \\
\hline Brazil & 17 & 0.57 & $\begin{array}{l}\text { France, Italy, South } \\
\text { Korea }\end{array}$ & 1 & 0.03 \\
\hline NA & 17 & 0.57 & Germany, Estonia & 1 & 0.03 \\
\hline Serbia & 16 & 0.53 & Germany, Netherlands & 1 & 0.03 \\
\hline Turkey & 16 & 0.53 & Germany, Norway & 1 & 0.03 \\
\hline South Korea & 15 & 0.5 & Israel, USA & 1 & 0.03 \\
\hline Singapore & 14 & 0.47 & Japan, Indonesia & 1 & 0.03 \\
\hline Sweden & 14 & 0.47 & Jordon & 1 & 0.03 \\
\hline Indonesia & 12 & 0.4 & Lithuania & 1 & 0.03 \\
\hline Hong Kong & 11 & 0.37 & Malaysia & 1 & 0.03 \\
\hline Nigeria & 11 & 0.37 & Malta & 1 & 0.03 \\
\hline Luxembourg & 10 & 0.33 & Moldova & 1 & 0.03 \\
\hline Northern Ireland, UK & 10 & 0.33 & Netherlands, Germany & 1 & 0.03 \\
\hline
\end{tabular}




$\begin{array}{lrrlll}\text { Republic of Ireland } & 10 & 0.33 & \text { Norway, Germany } & 1 & 0.03 \\ \text { Portugal } & 9 & 0.3 & \text { Poland, Germany } & 1 & 0.03 \\ \text { Croatia } & 8 & 0.27 & \text { Sharjah } & 1 & 0.03 \\ \text { Pakistan } & 8 & 0.27 & \text { Slovak Republic } & 1 & 0.03 \\ \text { UAE } & 8 & 0.27 & \text { South Africa } & 1 & 0.03 \\ \text { Finland } & 6 & 0.2 & \text { Sweden, Australia } & 1 & 0.03 \\ \text { Iran } & 6 & 0.2 & \text { Switzerland, Canada, } & 1 & 0.03 \\ \text { Korea } & 6 & 0.2 & \text { Switzerland, Poland } & 1 & 0.03 \\ \text { Taiwan } & 6 & 0.2 & \text { Thailand } & 1 & 0.03 \\ \text { Bosnia and } & 5 & 0.17 & \text { Turkey, UK } & 1 & 0.03 \\ \text { Herzegovina } & 5 & 0.17 & \text { UK, Belgium } & 1 & 0.03 \\ \text { Slovakia } & 4 & 0.13 & \text { UK, Italy } & 1 & 0.03 \\ \text { Germany, } & 5 & 0.13 & \text { UK, Norway } & 1 & 0.03 \\ \text { Switzerland } & 4 & 0.13 & \text { UK, USA } & 1 & 0.03 \\ \text { Greece } & 0.1 & \text { Ukraine } & 1 & 0.03 \\ \text { Netherlands, USA } & 4 & 0.1 & \text { USA, Chile } & 1 & 0.03 \\ \text { Canada } & 3 & 0.07 & \text { USA, UK } & 1 & 0.03 \\ \text { Colombia } & 3 & 0.07 & \text { Wales } & 1 & 0.03 \\ \text { Australia, UK } & 2 & & & & 1 \\ \text { Austria, Switzerland } & 2 & & \end{array}$

